\title{
DESENVOLVIMENTO DE UM APLICATIVO COMPUTACIONAL PARA A RECOMENDAÇÃO DE ADUBAÇÃO E CALAGEM PARA A CULTURA DO MILHO (Zea mays L.) NO ESTADO DO MARANHÃO, BRASIL
}

\author{
Luis Felipe Rodrigues de Aquino Sousa ${ }^{1}$; Wilson Araújo da Silva² ${ }^{2}$ Ricardo Viera Silva ${ }^{3}$ \\ ${ }^{1}$ Discente do curso de Agronomia da Universidade Estadual do Maranhão, Centro de Estudos Superiores de Imperatriz, \\ luis.felipe@msn.com \\ ${ }^{2}$ Eng. Agr., Prof. D.Sc, Adjunto da Universidade Estadual do Maranhão, Centro de Estudos Superiores de Imperatriz, , \\ wilsonufrrj@yahoo.com \\ ${ }^{3}$ Discente do curso de Agronomia da Universidade Estadual do Maranhão, Centro de Estudos Superiores de Imperatriz, \\ rickvieira_5@hotmail.com
}

\begin{abstract}
RESUMO: Aumentar a produção de grãos, para suprir a necessidade de alimento da população que cresce a cada dia, é um grande desafio. Para isso é importante que se utilize praticas que maximize a produtividade agrícola, bem como o manejo da adubação e calagem. A recomendação destes insumos seguem tabelas oficiais de acordo com sua região, assim muitas dessas recomendações é feita de maneira equivocada, devido à aplicabilidade regional. Desta forma é necessário que se tenha um sistema que possa superar essas barreiras, e que tais recomendações tenha o máximo critério técnico, clareza, responsabilidade no uso racional de fertilizantes e corretivos, além e maximizar a renda líquida dos produtores rurais. Para tanto, desenvolveu-se um aplicativo computacional, onde o mesmo foi estruturado utilizando o Excel 2010 e funções lógicas. Este aplicativo é usado para recomendar fertilizantes e corretivos especificamente para cultura do milho (Zea mays L.) e realizar a classificação do solo através de analise granulométrica, para o Estado do Maranhão. Após a análise dos resultados foi possível verificar que o aplicativo construído se mostrou eficiente quando comparado com outros aplicativos existentes no mercado.
\end{abstract}

PALAVRAS-CHAVE: corretivos, critério técnico, fertilizantes.

\section{DEVELOPMENT OF A COMPUTER APPLICATION FOR A RECOMMENDATION OF LIME AND FERTILIZER FOR CORN (Zea mays L.) AS MARANHÃO, BRAZIL}

\begin{abstract}
Increase grain production to meet the need for food of the population that grows every day is a challenge. For this it is important to use practices that maximize agricultural productivity, as well as the management of fertilization and liming. The recommendation of these inputs is following tables according to your area, so many of these recommendations is done wrongly, due to regional applicability. Thus it is necessary to have a system that can overcome these barriers, and that such recommendations have as much technical criteria, clarity, and responsibility in the rational use of fertilizers and, in addition to maximizing the net income of farmers. For this purpose, we developed software, where it was structured using Excel 2010 and logic functions. This application is used for fertilizers and recommend specifically for corn (Zea mays L.) and perform the classification of soil through particle size analysis for the Maranhão State. After the analysis of results was possible concluded your efficiency when compared with others commercials applications.
\end{abstract}

KEY-WORDS: correction, fertilizers, technical criterion.

Agroecossistemas, v. 3, n. 1, p. 36-40, 2011 


\section{INTRODUÇÃO}

O milho (Zea mays L.) é largamente cultivado e consumido em todos os continentes, com produção de cerca de 600 milhões de toneladas. Os Estados Unidos, a China e o Brasil são os maiores produtores mundiais. A produtividade brasileira tem crescido sistematicamente, passando de 1.665 $\mathrm{kg} \mathrm{ha}^{-1}$, em 1980, para $3.600 \mathrm{~kg} \mathrm{ha}^{-1}$, em 2009 (CANCELLIER, 2011).

Um dos componentes mais importantes para esse desenvolvimento da agricultura, principalmente no que diz respeito ao aumento da produtividade agrícola, sem esquecer os outros fatores de produção, foi a pesquisa em fertilizantes e as novas inovações cientificas e tecnológicas que permitiram o uso eficiente de corretivos e fertilizantes na agricultura brasileira (LOPES, 2007).

Assim esses insumos devem ser usados de forma eficiente, definindo-se que nutriente deve ser fornecido à planta, em quantidade e na época adequada. Dessa forma, para uma adequada recomendação de adubação é necessário identificar quais são os nutrientes limitantes ao crescimento e desenvolvimento das culturas (FILHO, 2010).

Deste modo, o uso racional de adubos e corretivos na agricultura, contribui para a otimização da renda líquida dos produtores rurais, além de minimizar os impactos sobre o meio ambiente, pois reduz a contaminação do solo e dos recursos hídricos devido à utilização indiscriminada.
O objetivo deste trabalho foi desenvolver um aplicativo computacional de recomendação de adubação e calagem para cultura do milho, baseado nos dados contidos no Manual de Recomendações de fertilizantes para o Estado do Maranhão. Assim maximizando a renda líquida do produtor rural e o uso correto de fertilizantes e corretivos.

\section{MATERIAL E MÉTODOS}

O aplicativo foi desenvolvido no Laboratório de Agronomia da Universidade Estadual do Maranhão / Centro de Estudos Superiores de Imperatriz. Utilizando o Microsoft Excel, foi criado uma planilha que a partir dos valores encontrados na analise de solo, é capaz de recomendar fertilizantes, corretivo e realizar a classificação textural do solo.

A recomendação de fertilizantes é baseado a partir de informações contidas no Manual de Recomendações de fertilizantes para o Estado do Maranhão (ARAÚJO, 1979), utilizando funções lógicas "SE" (retornará um valor se uma condição for considerada VERDADEIRO e um outro valor se essa condição for considerada FALSO) e "E" ( Retornará VERDADEIRO se todos os seus argumentos forem avaliados como VERDADEIRO e retornará FALSO se um ou mais argumentos forem avaliados como FALSO), os valores das tabelas de referências de níveis de nutrientes de Fósforo e Potássio serão selecionados em função dos níveis 
encontrados na análise de solo, permitindo classificar os teores dos nutrientes em baixo, médio ou alto. Após esta etapa, a rotina exibirá a recomendação de adubação em função da tabela oficial da cultura do milho.

A determinação da necessidade de calagem parti do critério de neutralização do alumínio e/ou elevação dos teores de cálcio e magnésio, usando as Equações 1 e 2 respectivamente.

1) $\mathrm{NC}(\mathrm{t} / \mathrm{ha})=\mathrm{mmol}_{\mathrm{c}} \mathrm{Al}^{3+} \cdot \mathrm{dm}^{3} \times 2$

2) $\mathrm{NC}(\mathrm{t} / \mathrm{ha})=\left(2-\mathrm{mmol}_{\mathrm{c}} \mathrm{Ca}^{++}+\mathrm{Mg}^{++}\right) \mathrm{x}$

em que,

$\mathrm{NC}=\mathrm{t} / \mathrm{ha}$ de calcário a ser aplicado, considerando a camada de $0-20 \mathrm{~cm}$.

$\operatorname{mmol}_{\mathrm{c}} \mathrm{Al}^{3+} \cdot \mathrm{dm}^{3}=$ revelado pela análise de solo.

$\mathrm{mmol}_{\mathrm{c}} \mathrm{Ca}^{++}+\mathrm{Ma}^{++}=$revelado pela análise de solo.

Onde o maior valor obtido será atribuído para recomendação da calagem, e que será multiplicado por (100/PRNT), para descontar a quantidade de calcário perdido pelo potencial de neutralização total.

A classificação da textura do solo será realizada com base nos valores em porcentagem da análise granulométrica do solo utilizando-se o triângulo textural (SANTOS, 2005). A partir dos valores da análise granulométrica, a função lógica "SE" vai realizar um teste lógico que retornará a duas possibilidades, verdadeiro ou falso, até encontrar o valor correspondente do triângulo textural.

Os resultados de recomendação de adubação e calagem obtidos com o aplicativo desenvolvido serão comparados com outros aplicativos existente no mercado: o Cadub 2.0 e AgroUtil. Para tanto usará valores de macronutrientes trocáveis expressos em mmol $_{\mathrm{c}} / \mathrm{dm}^{3}$, fornecidos para ambos aplicativos, sendo ele: $\mathrm{Ca}, \mathrm{Mg}, \mathrm{Al}, \mathrm{Ca}+\mathrm{Mg}$, e os valores respectivamente: 1,$5 ; 0,4 ; 0,2 ; 1,9$, e valores de $\mathrm{P}$ e $\mathrm{K}$ em $\mathrm{mg} / \mathrm{dm}^{3}, 1$ e 40 respectivamente, e PRNT igual a $80 \%$ para recomendação de calagem. Para classificação textural do solo elevou em consideração os valores da análise granulométrica do solo em porcentagem, onde os valores de areia, argila e silte foram respectivamente: $77,15,8$.

O usuário poderá realiza a recomendação preenchendo as células da planilha com os dados correspondente obtidos na análise de solo, onde estas células da planilha que necessitam de preenchimento não estão bloqueadas para edição e são indicadas pela cor cinza, as demais células não são editáveis e são indicadas pela cor branca.

O laudo gerado com as recomendações de adubação, calagem e a classificação textural do solo poderá ser impresso e/ou salvo na extensão "xlsx", para consulta e assinatura do técnico responsável e entrega para o solicitante.

Agroecossistemas, v. 3, n. 1, p. 36-40, 2011 


\section{RESULTADOS E DISCUSSÃO}

As recomendações geradas pelo aplicativo desenvolvido foi comparado com as recomendações do AgroUtil e Cadub 2.0, e constatou-se que tanto para recomendação de adubação como para de calagem calculadas pelos aplicativos, houve grande diferença para ambas recomendações, salvo para calagem onde a recomendação do AgroUtil foi idêntica a recomendação do aplicativo desenvolvido, conforme apresentado na Figura 1.

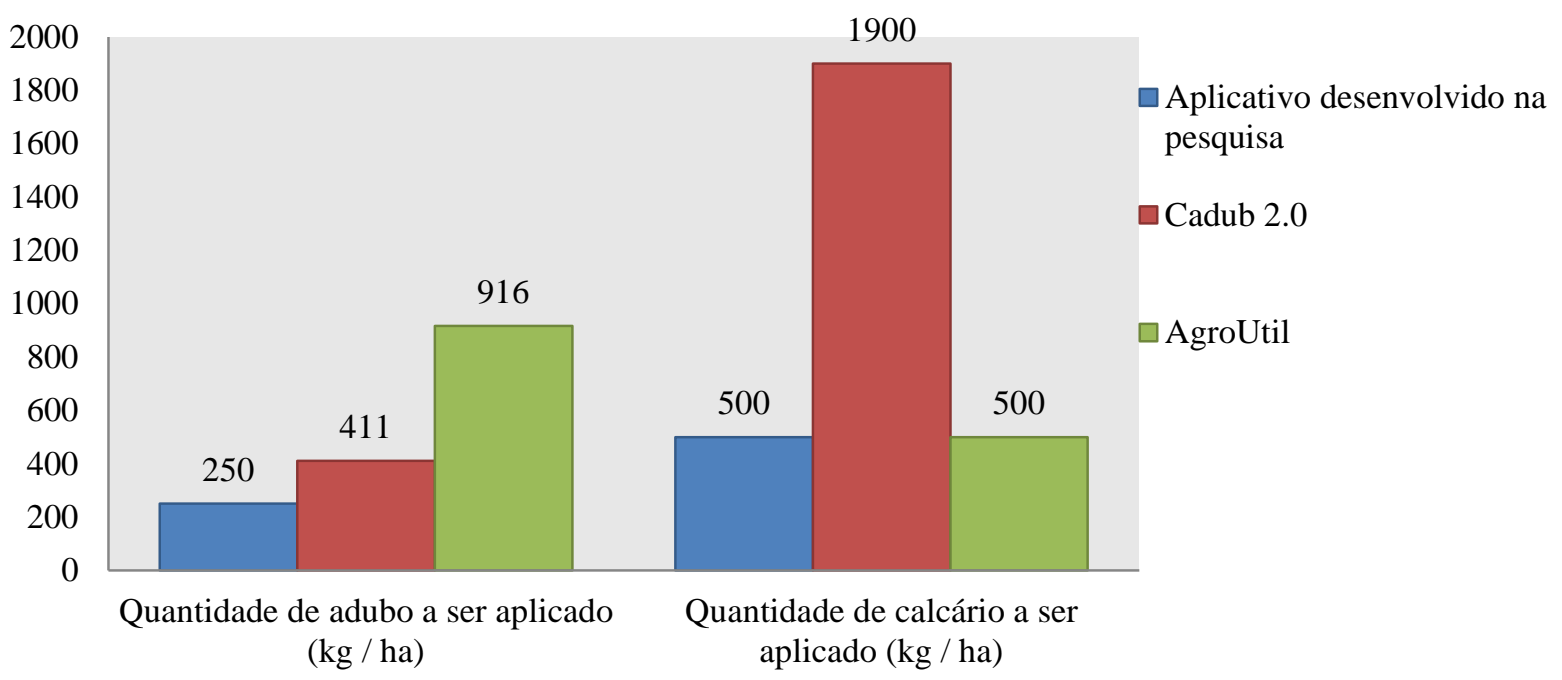

Figura 1:Diferença da quantidade de adubos e corretivo recomendada pelos aplicativos.

Além disso, o aplicativo desenvolvido foi único dos três aplicativos que realizou com êxito a classificação do solo com base nos valores da analise granulométrica do solo analisado, classificando o mesmo em Franco arenoso, o que deixa a recomendação mais detalhada, fornecendo subsídios para a escolha das fontes de nutrientes.

Os aplicativos em questão apresentam grande diferença das interfaces com o usuário, o Cadub 2.0 por exemplo é necessário realizar vários cadastros em vários ambientes diferentes, o AgroUtil também possui algumas interfaces com o usuário e não possibilita a expedição de um laudo pronto, já o aplicativo desenvolvido possui uma única interface o que agiliza a recomendação, o laudo já esta pronto na planilha do Excel, faltando apenas o preenchimento dos campos indicados e a recomendação é feita instantaneamente.

\section{CONCLUSÕES}

Com base na análise dos resultados obtidos foi possível estabelecer as seguintes conclusões: $\mathrm{O}$ aplicativo desenvolvido apresentou-se como uma ferramenta eficiente na recomendação de adubos e corretivos para 
a cultura do milho (Zea mays L.); Possibilitou a classificação da textura do solo facilitando a escolha das fontes de nutrientes; Permitiu uma maior e economia na recomendação de adubos e corretivos, contribuindo para a maximização da produção agrícola.

\section{REFERÊNCIAS}

ARAÚJO, F.S. Recomendações de fertilizantes para o Estado do Maranhão. São Luís: EMATER- MA, 1979.

CANCELLIER, L. L. Desempenho de populações de milho para produção de grãos e consumo in natura no Estado de Tocantins. Revista Ciência Agronômica. Fortaleza, v. 42, n. 2, p. 542-553, abr-jun, 2011. Disponível em:<http://www.ccarevista.ufc.br/seer/inde x.php/ccarevista/article/viewFile/1082/573> Acesso em: 13 mai. 2011.

FILHO, J. F. et al. Avaliação da fertilidade química dos solos nos sistemas de produção em assentamentos rurais nos municípios de Tamarana, Londrina e Ortigueira (PR), Brasil. In: Congresso da Sociedade Brasileira de Sistemas de Produção, 8., 2010, São Luís, MA. Anais...VIII Congresso da Sociedade Brasileira de Sistemas de Produção/UEMA, Mestrado em Agroecologia: EMBRAPA. São Luís: UEMA, 2010. p.463-478. 1 CD-ROM.

LOPES, A. S.; GUILHERME, L. R. G. Fertilidade do solo e produtividade agrícola. In: NOVAIS, R.F. et al., Eds. Fertilidade do
Solo. Viçosa: Sociedade Brasileira de Ciência do Solo, 2007. Cap. 1, p.1-64.

SANTOS, R. D. et al. Manual de descrição e coleta de solo no campo. 5. ed. Viçosa: Sociedade Brasileira de Ciência do Solo, 2005. 100 p. 